\title{
ADAPTAÇÃO TRANSCULT URAL E VALIDAÇÃO DE UM QUESTIONÁRIO DE CONHECIMENTO SOBRE INSUFICIÊNCIA CARDÍACA PARA ENFERMEIROS
}

\author{
Cristini KLEIN ${ }^{a}$, G raciele Fernanda da Costa $\mathrm{LIN} \mathrm{CH}^{\mathrm{b}}$, E miliane N ogueira de SOU ZAc

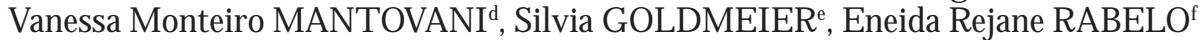

\begin{abstract}
RESUM 0
E studo com objetivo de realizar a adaptação transcultural, assim como verificar a validade de conteúdo e a estabilidade do $\mathrm{N}$ urses' Knowledge of $\mathrm{H}$ eart Failure $\mathrm{E}$ ducation Principles para avaliar $\mathrm{O}$ conhecimento de enfermeiros brasileiros sobre insuficiência cardíaca. 0 processo de adaptação transcultural constituiu-se da tradução, síntese, retrotraducão, revisão por um comitê e pré-teste. Foram avaliadas as propriedades psicométricas: validade de conteúdo (face), fidedignidade por meio da consistência interna (Alfa de Cronbach) e estabilidade (Coeficiente Kappa). A pós adaptação transcultural, o instrumento foi aplicado a 54 enfer meiros (27 de um hospital especializado em cardiologia e 27 de hospital geral). 0 A lfa de Cronbach foi de 0,7. A s questões de númer os 4, 5 e 11 apresentaram coeficiente Kappa inferior ou igual a 0,4, e as demais questões apresentaram Kappa superior ou igual a 0,7. E ste questionário foi validado e mostrou-se adequado para avaliar o conhecimento desse grupo de profissionais.
\end{abstract}

D escritores: Insuficiência cardíaca. Conhecimento. Q uestionários. E studos de validação.

\section{RESUMEN}

E studio que propone realizar la adaptación transcultural y comprobar la autoridad del contenido y la estabilidad del $\mathrm{N}$ urses Knowledge of $H$ eart $F$ ailure $E$ ducation $P$ rinciples para evaluar el conocimiento de enfermeros brasileños sobre insuficiencia cardíaca. E I proceso de adaptación transcultural se constituye de traducción, síntesis, retrotraducción, revisión por un comité y pre test. F ueron evaluadas las propiedades psicométricas: validad de contenido (fase), confiabilidad a través de consistencia interna (Alfa de Cronbach) y estabilidad (Coeficiente Kappa). T ras la adaptación transcultural, el instrumento fue aplicado a 54 enfermeros, (27 dehospital especializado en cardiología y 27 de hospital general). A lfa deCronbach fuede 0,7. L as cuestiones número 4,5 y 11 presentaron coeficienteK appa inferior o igual a 0,4 - las demás cuestiones presentaron Kappa superior o igual a 0,7. E ste cuestionario es válido y se mostró adecuado para evaluar el conoci miento de ese grupo de profesionales.

Descriptores: Insuficiencia cardíaca. Conocimiento. Cuestionarios. E studios de validación.

Título: A daptación transcultural y validad de un cuestionario de conocimiento sobreinsuficiencia cardiaca para enfermeros.

\section{ABST RACT}

This study aims to perform the cross-cultural adaptation and to verify the content validity and stability of $\mathrm{N}$ urses' Knowledge of $\mathrm{H}$ eart $\mathrm{F}$ ailureE ducation $\mathrm{P}$ rinciples to eval uate what $\mathrm{B}$ razilian nurses know of heart failure Theprocess of cross-cultural adaptation involved translation, synthesis, back-translation, committee's proofreading and pre test. T hefollowing psychometric properties w ere assessed: content validity (face), reliability through internal consistency (Cronbach's A Ipha) and stability (Kappa coefficient). A fter the cross-cultural adaptation, the instrument was applied to 54 nurses (27 from a cardi ol og y hospital and 27 from a general hospital). T heCronbach's A Ipha was 0.7. T hequestions 4, 5, and 11 presented a Kappa coefficient less than or equal to 0.4 , and further questions presented a Kappa coef ficient superior or equal to 0.7 . T his questionnaire was validated and proved to be adequate to assess the know ledge of this group of professionals.

Descriptors: H eart failure Knowledge. Questionnaires. Validation studies

$T$ itle: Cross-cultural adaptation and validation of a questionnaire on what nurses know of heart failure.

\footnotetext{
a M estre em Biologia M olecular e Celular, Especialista em Enfermagem em Cardiologia, Enfermeira Assistencial do Serviço de Enfermagem em Terapia Intensiva do Hospital Clínicas de Porto Alegre (HCPA), Porto Alegre, Rio G rande do Sul, Brasil.

- M estre em Enfermagem, D outoranda do Programa de Pós-G raduação em Enfermagem da U niversidade Federal do Rio Grande do Sul (UFRGS), Bolsista CAPES, Porto Alegre, Rio Grande do Sul, Brasil.

' D outora em Ciências da Saúde, Professora do Programa de Pós-G raduação L ato Sensu em Enfermagem em Cardiologia do Instituto de Cardiologia - Fundação U niversitária de Cardiologia (IC-FUC) e da U niversidade Federal de Ciências da Saúde de Porto Alegre, Rio Grande do Sul, Brasil.

d Enfermeira A ssistencial do H ospital São Francisco Sul, Porto Alegre, Rio G rande do Sul, Brasil.

e D outora em Ciências da Saúde: Cardiologia, Professora do Programa de Pós-G raduação L ato Sensu em Enfermagem em Cardiologia do IC-FUC, Porto Alegre, Rio Grande do Sul, Brasil.

f D outora em Ciências Biológicas: Fisiologia, Professor A djunto da Escola de Enfermagem e dos Programas de Pós-G raduação em Enfermagem e em Ciências da Saúde: Cardiologia e Ciências Cardiovasculares da UFRGS, Professora do Programa de Pós-G raduação Lato Sensu em E nfer magem em Cardiologia do I C-F U C, Coordenadora da Clínica de Insuficiência Cardíaca do H CPA, Bolsista de Produtividade em Pesquisa do CN Pq, Porto Alegre, Rio Grande do Sul, Brasil.
} 


\section{INT RODUÇÃO}

0 prognóstico de pacientes com insuficiência cardíaca (IC) depende da combinação de estratégias de tratamento que incluem tanto os aspectos farmacológicos, que visam reduzir morbidade e mortalidade, como a abordagem não farmacológica, considerada importante ferramenta na redução de eventos como crises de descompensação e readmissões ${ }^{(1,2)}$.

A última meta-análise sobre a abordagem multidisciplinar em IC reforça benefícios no seguimento destes pacientes quanto à redução de readmissões e, portanto, diminuição de custos com internações, além de menores taxas de mortalidade associadas a essa síndrome ${ }^{(3)}$.

D entro do contexto da abordagem não farmacológica, um programa contínuo e sistemático de educação sobre IC, uso regular das medicações, restrição de sal e de líquidos na dieta, atividade física regular, controle de peso e alterações no estilo de vida são essenciais para estes pacientes ${ }^{(2,4)}$. Estratégias de educação e orientações para 0 autocuidado de pacientes com IC exigem, por parte dos enfermeiros, um conhecimento consistente e atualizado.

Considerando o conhecimento no manejo de pacientes com IC, poucos estudos na literatura têm investigado o conhecimento dos enfermeiros a respeito do assunto(5,6). Em 2002, um estudo norteamericano avaliou, em cinco hospitais, o conhecimento de 300 enfermeiros em relação à IC. N esse estudo, foi aplicado um questionário composto de 20 questões que incluíam abordagens farmacológicas e não farmacológicas no tratamento desses pacientes. Os resultados do estudo evidenciaram o conhecimento insuficiente para o manejo de pacientes com IC por parte dos enfermeiros avaliados $^{(5)}$. U m estudo semelhante, utilizando a mesma metodologia, avaliou 51 enfermeiros, demonstrando que os profissionais também apresentaram um conhecimento insatisfatório no manejo desses pacientes ${ }^{(6)}$.

Tendo em vista as altas taxas de mortalidade, inter nações e re-internações hospitalares, e as consequentes limitações impostas a esses pacientes, tem-se estabelecido programas de educação sobre IC e autocuidado a portadores e cuidadores. 0 gerenciamento de pacientes por uma equipe multidisciplinar, com enfermeiros atuantes no manejo da IC, principalmente em ambiente hospita- lar, tem trazido benefícios para melhora da adesão ao tratamento, redução de readmissões e diminuição dos custos intra-hospitalares ${ }^{(7,8)}$. N o entanto, 0 campo de investigação quanto ao preparo de enfermeiros para a realização dessa abordagem permanece inexplorado no cenário brasileiro. Neste contexto, este estudo teve por objetivo realizar a adaptação transcultural e validação do questionário $\mathrm{N}$ urses' Knowledge of $\mathrm{H}$ eart Failure $\mathrm{E}$ ducation P rinciples para avaliar o conhecimento de enfermeiros brasileiros sobre IC.

\section{MÉTODOS}

T rata-se de um estudo metodológico(9), realizado com enfermeiros de um hospital especializado em cardiologia e enfermeiros de um hospital geral, ambas as instituições no E stado do Rio G rande do Sul. $O$ instrumento original N urses's Knowledge of $\mathrm{H}$ eart Failure (NKHF)(5) foi publicado em 2002. Foi solicitado a autorização da autora $\mathrm{N}$ ancy M. Albert para realizar o processo adaptação transcultural e validação. Participaram deste estudo 54 enfermeiros que trabal havam na unidade de internação clínica, cirúrgica, emergência e unidade de tratamento intensivo (UTI). A sel eção da amostra foi realizada de forma aleatória simples. Os enfermeiros com menos de quatro meses de atividade nas instituições foram excluídos.

\section{N urses's Knowledge of Heart Failure - Questio- nário de Conhecimento de Enfermeiros sobre Insuficiência Cardíaca (Q-CENIC) e Processo de Adaptação T ranscultural e Validação}

0 instrumento original contém 20 questões que incluem conhecimento sobre dieta (restrição de sódio e de líquidos), controle de peso, sinais e sintomas da IC e tratamento, total izando 20 questões. 0 processo de tradução, adaptação transcultural e validação de conteúdo incluiu as seguintes etapas: tradução inicial, síntese, retrotradução, avaliação da tradução por um comitê de juízes (três enfermeiras especialistas em cardiologia, sendo duas docentes de graduação em E nfermagem, com domínio da língua inglesa e a pesquisadora que possui ampla experiência em IC e um profissional com formação em linguística), avaliação semântica, pré-teste, e verificação das propriedades psicométricas (Alfa de Cronbach e Coeficiente de $\mathrm{K}$ appa) ${ }^{(10)}$. A tradução inicial foi realizada por dois 
professores de inglês, brasileiros. A pós, os dois tradutores reuniram-se com um dos pesquisadores para e produzir um único questionário em português (síntese) a partir das versões traduzidas. A versão de síntese em português foi traduzida para 0 inglês (retrotradução) por outros dois professores de língua inglesa cuja língua materna é o português. Esses profissionais desconheciam os objetivos da pesquisa. Para avaliação das equivalências, as traduções for am aval iadas pel os membros do comitê de juízes, os quais receberam a versão original do NKHF, a versão de síntese, e de retrotradução. A pós a revisão e discussão, elaborou-se uma versão de consenso, sendo que as modificações foram realizadas quando pelo menos dois dos membros concordavam com a alteração.

Para validação semântica do NKHF foi realizada uma discussão com enfermeiros respondentes, procurando identificar ambiguidades e dificuldades de compreensão, verificar a adequação dos itens e obter críticas e sugestões de mel horia. A pós foi realizado o préteste com 10 enfermeiros de ambas as instituições com o objetivo de testar o questionário adaptado. D urante o pré-teste, obtiveramse o máximo de informações sobre a compreensão do entrevistado referente aos itens questionados. As pesquisadoras analisaram e discutiram novamente as questões, e embasadas nas sugestões dos sujeitos que participaram do pré-teste realizaram al gumas alterações no N KHF para seu melhor entendimento. $\mathrm{N}$ este trabal ho optou-se pela verificação da fidedignidade, por meio do alfa de Cronbach e, para verificação da estabilidade utilizou-se o Coeficiente K appa. Para a estabilidade foi real izado um teste-reteste com 28 enfermeiros, nas mesmas circunstâncias, após duas semanas do primeiro teste.
A pós as etapas de tradução e adaptação, 0 instrumento ficou com 13 questões do instrumento original e três incluídas pelo comitê de especial istas, o qual foi validado em uma amostra do tipo não probabilística de 54 enfermeiros de dois hospitais.

0 projeto foi aprovado por Comitê de Ética sob o número 3811/06, e todos os enfermeiros incluídos no estudo leram e assinaram o T ermo de Consentimento Livre e E sclarecido.

\section{Análise estatística}

Os dados foram inseridos em uma planilha do Programa Excel for W indows. As análises foram realizadas utilizando-se 0 pacote estatístico Statistical Package for Social Sciences (SPSS) 15.0. Para avaliar a fidedignidade do instrumento foi utilizado o A lfa de Cronbach e para a estabilidade o Coeficiente K appa. As variáveis categóricas foram expressas com percentual ou valor absoluto; as contínuas com média \pm desvio padrão ou mediana e intervalo interquartil 25 e 75, conforme seguiram ou não a distribuição normal. Para o cálculo amostral foi utilizado o teste t, com poder estatístico de $95 \%$ e alfa de $5 \%$ necessitando a inclusão de 54 enfermeiros. Foram consideradas significativas as relações com $P \leq 0,05$.

\section{RESULT AD OS}

\section{Validação do instrumento Q-CE NIC}

O Quadro 1 apresenta os itens do questionário original e sua respectiva adaptação para o Brasil. Foram excluídas sete questões $(8,10,16-20)$ e inseridas três, descritos nas últimas linhas do Quadro 1.

\begin{tabular}{|l|l|}
\hline \multicolumn{1}{|c|}{ Q uestionário original } & \multicolumn{1}{|c|}{ Q uestionário adaptado e validado } \\
\hline $\begin{array}{l}\text { 1. Patients with H F should drink plenty of fluids each day. } \\
\text { (F) }\end{array}$ & $\begin{array}{l}\text { Pacientes com IC devem tomar bastante líquido todos } \\
\text { os dias. (F) }\end{array}$ \\
\hline $\begin{array}{l}\text { 2. As long as no salt is added to foods, there are no dietary } \\
\text { restrictions for patients with H F. (F) }\end{array}$ & $\begin{array}{l}\text { Contanto que não se adicione sal aos alimentos, não há } \\
\text { restrições na dieta para pacientes com IC. (F) }\end{array}$ \\
\hline $\begin{array}{l}\text { 3. Coughing and nausea/ poor appetitearecommon symptoms } \\
\text { of advanced H F. (T ) }\end{array}$ & $\begin{array}{l}\text { Tosse, náuseas e diminuição do apetite são sintomas co- } \\
\text { muns da IC num estágio mais avançado. (V) }\end{array}$ \\
\hline $\begin{array}{l}\text { 4. P atients with H F should decreaseactivity and most forms } \\
\text { of active exercise should be avoided. (F) }\end{array}$ & $\begin{array}{l}\text { Pacientes com IC devem reduzir as atividades e a maio- } \\
\text { ria das formas de exercícios ativos deve ser evitada. (F) }\end{array}$ \\
\hline
\end{tabular}


Continuação.

\begin{tabular}{|c|c|}
\hline Questionário original & Questionário adaptado e validado \\
\hline $\begin{array}{l}\text { 5. If the patient gains more than } 3 \text { pounds in } 48 \text { hours without } \\
\text { other HF symptoms, they should not be concerned. (F ) }\end{array}$ & $\begin{array}{l}\text { Se o paciente apresentar acréscimo de mais de } 1.400 \mathrm{Kg} \\
\text { em } 48 \text { horas sem outros sintomas de IC, este aumento } \\
\text { de peso não deve ser considerado. (F) }\end{array}$ \\
\hline $\begin{array}{l}\text { 6. Sw elling of theabdomen may indicateretention of excess } \\
\text { fluid due to worsening H F. (T) }\end{array}$ & $\begin{array}{l}0 \text { abdome globoso pode indicar retenção de líquido de- } \\
\text { vido à piora da IC. (V) }\end{array}$ \\
\hline $\begin{array}{l}\text { 7. If patients take their medications as directed and follow } \\
\text { the suggested lifestylemodifications, their H F condition will } \\
\text { not return. (F ) }\end{array}$ & $\begin{array}{l}\text { Se o paciente tomar seus medicamentos corretamente e } \\
\text { seguir as orientações adequadas em relação ao "estilo } \\
\text { de vida" sugerido, poderá curar a IC. (F) }\end{array}$ \\
\hline $\begin{array}{l}\text { 8. W hen patients have aches and pains, aspirin and non- } \\
\text { steroidal anti-inflammatory drugs (N SAID slikeibuprofen) } \\
\text { should be recommended. (F) }\end{array}$ & Excluída. \\
\hline $\begin{array}{l}\text { 9. It is ok to use potassium-based salt substitutes (like "N o- } \\
\text { Salt" or "Salt S ense") to season food. (F) }\end{array}$ & Excluída. \\
\hline $\begin{array}{l}\text { 10. If patients feel thirsty, it is ok to remove fluid limits and } \\
\text { allow them to drink. (F) }\end{array}$ & $\begin{array}{l}\text { Excluída (conteúdo presente na questão } 1 \text { do instru- } \\
\text { mento original). }\end{array}$ \\
\hline $\begin{array}{l}\text { 11. If a patientadds extra pillows at night to relieveshortness } \\
\text { of breath, this does not mean that the H F condition has } \\
\text { worsened. (F) }\end{array}$ & $\begin{array}{l}\text { Se o paciente dormir com mais que um travesseiro à } \\
\text { noite para não sentir falta de ar, não significará que seu } \\
\text { estado de IC piorou. (F) }\end{array}$ \\
\hline $\begin{array}{l}\text { 12. If a patient wakes up at night with difficulty breathing, } \\
\text { and the breathing difficulty is relieved by getting out of bed } \\
\text { and moving around, this does not mean that the HF condition } \\
\text { has w orsened. (F) }\end{array}$ & $\begin{array}{l}\text { Se um paciente se acorda à noite com dificuldade para } \\
\text { respirar, e essa dificuldade é aliviada ao sair da cama e } \\
\text { caminhar, isso não significa que a condição da IC tenha } \\
\text { piorado. (F) }\end{array}$ \\
\hline $\begin{array}{l}\text { 13. L ean deli meats are an acceptable food choice as part of } \\
\text { the patient's diet. (F ) }\end{array}$ & $\begin{array}{l}\text { Car nes magras processadas são uma escol ha al imentar } \\
\text { aceitável como parte da dieta do paciente. (F) }\end{array}$ \\
\hline $\begin{array}{l}\text { 14. Oncethepatient's H F symptoms aregone, there is no need } \\
\text { for obtaining daily w eights. (F) }\end{array}$ & $\begin{array}{l}\text { U ma vez que os sinais de IC desaparecerem, não é mais } \\
\text { necessário a pesagem diária (do paciente). (F) }\end{array}$ \\
\hline $\begin{array}{l}\text { 15. W hen assessing weight results, today's weight should be } \\
\text { compared with the patient's weight from yesterday, not the } \\
\text { patient's ideal or "dry" weight. (F ) }\end{array}$ & $\begin{array}{l}0 \text { controle do peso diário deverá ser comparado com o } \\
\text { peso do dia anterior, e não com o peso ideal ou peso seco } \\
\text { do paciente. (F) }\end{array}$ \\
\hline $\begin{array}{l}\text { The following } 5 \text { statements reflect signs or symptoms that } \\
\text { patients may have. M ark "yes" (T) or "no" (F) to signify } \\
\text { that a patient should notify their H F physician of thesesigns } \\
\text { or symptoms: }\end{array}$ & \\
\hline $\begin{array}{l}\text { 16. B P recording of } 80 / 56 \text { without any H F symptoms. ( } \mathrm{N} \text { o/ } \\
\text { F ) }\end{array}$ & Excluída. \\
\hline $\begin{array}{l}\text { 17. Weight gain of } 3 \text { pounds in } 5 \text { days without symptoms. } \\
(Y e s / T)\end{array}$ & $\begin{array}{l}\text { Excluída (conteúdo presente na questão } 5 \text { do instru- } \\
\text { mento original). }\end{array}$ \\
\hline
\end{tabular}


Continuação.

\begin{tabular}{|c|c|}
\hline Questionário original & Q uestionário adaptado e validado \\
\hline $\begin{array}{l}\text { 18. Dizziness or lightheadedness when arising that } \\
\text { disappears within } 10-15 \text { minutes. ( } \mathrm{N} \text { o/ } \mathrm{F} \text { ) }\end{array}$ & Excluída \\
\hline 19. $N$ ew onset or worsening of fatigue. (Y es/ T ) & $\begin{array}{l}\text { Excluída. (conteúdo presente indiretamente nas ques- } \\
\text { tões: } 5,6,11,12 \text { e 14) }\end{array}$ \\
\hline $\begin{array}{l}\text { 20. N ew onset or worsening of leg weakness or decreased } \\
\text { ability to exercise. (Y } \mathrm{Y} / \mathrm{T} \text { ) }\end{array}$ & $\begin{array}{l}\text { Excluída. (conteúdo presente indiretamente nas ques- } \\
\text { tões: } 5,6,11,12 \text { e 14) }\end{array}$ \\
\hline-- & $\begin{array}{l}\text { Pacientes estáveis podem ser encorajados a manter sua } \\
\text { atividade sexual, fazendo os ajustes necessários para } \\
\text { evitar esforço em demasia e o surgimento de sintomas. } \\
\text { (V) }\end{array}$ \\
\hline-- & $\begin{array}{l}\text { Os pacientes com IC devem ser orientados, quando em } \\
\text { uso de nitratos, que só podem fazer uso de sildenafil } 24 \\
\text { horas após a suspensão do nitrato. (V) }\end{array}$ \\
\hline-- & $\begin{array}{l}\text { O uso correto das medicações e a adesão as medidas de } \\
\text { controle de dieta, peso, ingesta hídrica, podem evitar } \\
\text { crises de descompensações. (V) }\end{array}$ \\
\hline
\end{tabular}

Legenda: HF : heart failure; IC: insuficiência cardíaca; Y es/ T/ V = sim/ verdadeiro; $\mathrm{N}$ o/ F = não/ falso

Q uadro 1 - Avaliação da equivalência semântica entre 0 instrumento original em inglês e a versão final em português. Porto Alegre, RS, 2007.

Para avaliar a consistência interna do questionário, foi utilizado o Alfa de Cronbach, com resultado de 0,7 . E $m$ relação à estabilidade, as questões de números 4, 5 e 11 apresentaram coeficiente de Kappa inferior ou igual a 0,4 , sendo que as demais questões apresentaram Kappa superior ou igual a 0,7 .
Do total de enfermeiros selecionados para participar desde estudo, três não aceitaram, totalizando uma amostra de 54 enfermeiros, 27 de um hospital especializado em cardiologia e 27 de um hospital geral, com idade média de $31 \pm 6$ anos, sendo $48(92,6 \%)$ do sexo feminino. D ados demonstrados na T abela 1.

T abela 1 - Características dos enfermeiros avaliados de ambos os hospitais. Porto Alegre, RS, 2007.

\section{Características}

Idade*

Sexo (feminino)

E specialização em curso

E specialização completa

M estrado completo/ em cur so

T empo de formação superior ${ }^{\dagger}$

Tempo de trabal ho na instituição ${ }^{\dagger}$

U nidade inter nação clínica

U nidade internação cirúrgica

E mergência

U nidade terapia intensiva

\begin{tabular}{c}
$\begin{array}{c}\text { Todos } \\
\text { (n=54) }\end{array}$ \\
\hline $\mathbf{n}(\%)$ \\
\hline $31 \pm 6$ \\
$48(92,6)$ \\
$17(31,5)$ \\
$14(25,9)$ \\
$3(5,6)$ \\
$4,2(2,5-9)$ \\
$2(0,9-4,5)$ \\
$39(72,2)$ \\
$31(57,4)$ \\
$18(33,3)$ \\
$18(33,3)$
\end{tabular}

$\begin{gathered}\text { Especializado } \\ (\mathbf{n}=\mathbf{2 7})\end{gathered}$
$\mathbf{n}(\%)$
$32,1 \pm 7$
$23(85,2)$
$16(59,3)$
$8(29,6)$
$3(11,1)$
$4,5(2-9)$
$2(1-7)$
$18(66,7)$
$12(44,4)$
$9(33,3)$
$12(44,4)$

\begin{tabular}{ccc}
$\begin{array}{c}\text { G eral } \\
\text { (n=27) }\end{array}$ & P \\
\cline { 1 - 1 } $\mathbf{n ( \% )}$ & & \\
\hline $30 \pm 5$ & & 0,18 \\
$27(100,0)$ & & 0,11 \\
$21(77,8)$ & & 0,14 \\
$6(22,2)$ & & 0,14 \\
- & & 0,14 \\
$4(2,5-7)$ & & 0,61 \\
$2(0,8-4,5)$ & & 0,55 \\
$21(77,8)$ & & 0,54 \\
$19(70,4)$ & & 0,09 \\
$9(33,3)$ & & 0,10 \\
$6(22,2)$ & & 0,15
\end{tabular}

* Valores descritos como média \pm desvio padrão; ${ }^{\dagger}$ Valores descritos como mediana e intervalo interquartil 25 e 75 


\section{DISCUSSÃO}

N esse estudo objetivou-se adaptar e validar um questionário de conhecimento sobre o manejo da IC para uso no Brasil. A adaptação e validação de um instrumento para outro idioma é um processo complexo, no qual deve-se levar em conta aspectos técnicos, linguísticos e semânticos, em virtude das diferenças culturais. No presente estudo de validação foram excluídas, adaptadas e acrescidas al gumas questões visando mel hor ar o entendimento da população alvo. No que se refere às questões inseridas, salienta-se que as mesmas complementam os conhecimentos essenciais ao manejo clínico de pacientes com IC.

Tendo em vista que não existem métodos objetivos para mensurar precisamente a validade de conteúdo de um instrumento, este pode ser realizado por meio de um comitê de juízes/ especialistas ${ }^{(10)}$ como realizado no presente estudo, em que avaliaram a relevância de cada questão do questionário individualmente. Quando a questão é traduzida, mas não reporta atividades realizadas em uma determinada cultura, deve ser identificada uma situação similar para substituí-la $a^{(10)}$.

Os itens excluídos para a versão adaptada não se aplicavam à realidade brasileira ou ainda, continham informações contempladas em outras questões do questionário. A questão 10 do instrumento original não foi validada na sua forma literal, porém entendemos que seu conteúdo está contemplado na questão um do instrumento original. $\mathrm{Da}$ mesma forma, as questões de número 16 à 20 do instrumento original, apresentadas na forma de sinais e sintomas da IC, não foram validadas na sua forma literal, porém seu conteúdo está incluso nas demais questões do instrumento.

Outras duas questões inseridas são referentes a cuidados com atividade sexual em pacientes com IC, assunto este não abordado na escala original. Essas questões foram desenvolvidas conforme diretrizes nacionais e inter nacionais para manejo de pacientes com I $C^{(1,11)}$, de acordo com a concordância dos especialistas. E ssas questões apresentaram estabilidade excel ente.

Considerando que o instrumento adaptado apresentou A Ifa de Cronbach adequado para a versão final (com exclusões e inclusões), não é recomendado retirar os três itens que apresentaram baixo coeficiente de Kappa.
No estudo original do instrumento N urses's Knowledge of $H$ eart Failure não foram avaliadas as variáveis psicométricas ${ }^{(5)}$. N o entanto, 0 instrumento adaptado no presente estudo (Q-CE NIC) mostrou consistência inter na adequada com A Ifa de 0,7.

Em estudo recente que objetivou comparar as propriedades psicométricas da escala original na sua língua materna, utilizando na sua grade de resposta a análise dicotômica (verdadeiro ou falso), comparado a escala de Likert (5 graduações), mostrou um Alfa de Cronbach melhor para o uso da escala de Likert ${ }^{(12)}$. Porém para al cançar 0 alfa de Cronbach de 0,70 foi necessária a exclusão de três questões, as questões de número 15, 16 e 18. No Q-CE NIC a questão de número 15 mostrou estabilidade adequada, com Kappa > 0,5, e as questões de número 16 e 18 não foram avaliadas, pois de acordo com o consenso dos especialistas essas não estavam adequadas para realidade brasileira.

Visto o resultado do estudo anteriormente citado sugerimos um novo estudo na mesma população utilizando a escala de Likert e a dicotômica. D essa maneira, a comparação entre os resultados da análise psicométrica de ambas deve ser verificada para que então seja escolhida a mel hor forma de se avaliar o entendimento dos enfermeiros sobrealC.

\section{CONCLUSÕES}

Os resultados relativos à validação deste questionário apresentaram fidedignidade e estabilidade adequadas para a avaliação do conhecimento de enfermeiros sobre IC em nosso meio, expressos respectivamente pelo Alfa de Cronbach e coeficiente Kappa.

A aplicação de um questionário com questões específicas sobre IC aos enfermeiros, tendo em vista a cronicidade da doença e as el evadas taxas de readmissões permite verificar o entendimento que os profissionais têm acerca desta doença. Além disso, permite, a partir dos resultados obtidos, que estratégias sejam pensadas para promover melhores e mais efetivas ações de cuidado e educação para a saúde de forma a qualificar a assistência de enfermagem aos pacientes com IC.

\section{RE FE RÊ NCIAS}

1 Bocchi EA , M arcondes-Braga F G, Ayub-Ferreira SM , Rohde LE, Oliveira WA, A Imeida DR, et al. III Dire- 
triz Brasileira de Insuficiência Cardíaca Crônica. Arq Bras Cardiol. 2009;92(6 Supl 1):1-71.

2 Riegel B, M oser DK, Anker SD, Appel LJ, D unbar $\mathrm{SB}, \mathrm{G}$ rady $\mathrm{KL}$, et al. State of the science: promoting self-care in persons with heart failure: a scientific statement from the A merican Heart Association. Circulation. 2009;120(12):1141-63.

3 Holland R, Battersby J, H arvey I, L enaghan E, Smith J, Hay L. Systematic review of multidisciplinary interventions in heart failure. Heart. 2005;91:899-906.

4 Rabelo ER, A liti GB, Domingues FB, Ruschel KB, Brun AO. $O$ que ensinar aos pacientes com insuficiência cardíaca e porquê: o papel dos enfermeiros em clínicas de insuficiência cardíaca. Rev Latino-Am Enfermagem. 2007;15(1):165-70.

5 Albert NM, Collier S, Sumondi V, W ilkinson S, H ammel JP, Vopat L, et al. N urses' knowledge of heart failure education principles. H eart L ung. 2002;31(2): 102-12.

6 W ashburn SC, H ornberger CA, Klutman A, Skinner L. N urses' knowledge of heart failure education topics as report in a small Midwestern community hospital. Eur J Cardiovasc N urs. 2005;20(3):215-20.

7 Bocchi EA, Cruz F, Guimarães G, M oreira LF P, Issa VS, Ferreira SM A, et al. A Long-term Prospective Randomized Controlled Study U sing Repetitive
Education at Six-M onth Intervals and M onitoring for Adherence in $\mathrm{H}$ eart Failure Outpatients: The RE M A D HE Study T rial. Circ H eart F ail. 2008;1:11524.

$8 \mathrm{M}$ angini $\mathrm{S}$, Silveira $\mathrm{FS}$, Silva CP, G rativvol PS, Seguro LF BC, Ferreira SM A, et al. Insuficiência cardíaca descompensada na unidade de emergência de hospital especial izado em cardiologia. A rq B ras Cardiol. 2008; 90(6):433-40.

9 LoBiondo-W ood G, Haber J. D esenhos não-experimentais. In: LoBiondo-Wood G, H aber J, organizadores. Pesquisa em enfermagem: métodos, avaliação crítica e utilização. 4ạ ed. Rio de Janeiro: G uanabara Koogan; 2001. p. 110-21.

10 Beaton D, Bombardier C, Guillemin F, Ferraz M. Guidelines for the process of cross-cultural adaptation of self-report measures. Spine. 2000;25(24): 3186-91.

11 Rabelo ER, Aliti GB, Goldraich L, D omínguez FB, Clausell $N$, Rohde $L E$, et al. $M$ anejo não farmacológico de pacientes hospitalizados com insuficiência cardíaca em hospital universitário. Arq Bras Cardiol. 2006;87(3):352-8.

$12 \mathrm{H}$ art PL, Spiva L, Kimble LP. Nurses' knowledge of heart failure education principles survey: a psychometric study. J Clin Nurs. 2011;20(21/22): 3020-8.

\section{Endereço da autora / Dirección del autor / Author's address:}

Eneida Rejane Rabelo

Escola de E nfermagem

U niversidade Federal do Rio G rande do Sul

Rua São M anoel, 963, R io Branco

90620-110, Porto Alegre, RS

E - mail: eneidarabelo@gmail.com
Recebido em: 16/ 11/ 2010

A provado em: 08/ 09/ 2011 